\section{Extracción de flavonoides totales de la envoltura externa de cebolla roja (Allium cepa)}

\section{Extraction of total flavonoids from the outer covering of red: onion (Allium cepa)}

Anyuri Fajardo-Romero*, Álvaro Arroyo-Rivera*, Juan Sebastián Ramírez-Navas

\author{
* Químico, Programa de Química, Universidad Santiago de Cali. \\ ** Ingeniero Químico, PhD en Ingeniería, Profesor. GIEMA, Universidad Santiago de Cali - GIPAB. \\ Universidad del Valle, C.P. 25360, Cali, Colombia.
}

\section{Resumen}

El objetivo de este trabajo fue cuantificar los flavonoides totales, expresados en quercetina, de la envoltura externa de la cebolla roja. Las muestras de cebollas se adquirieron en el mercado ecológico campesino de Cali (Valle del Cauca). Los extractos metanólicos se realizaron usando agitación, ultrasonido y UVC (ultrasonido, vortex y centrifuga). Se emplearon muestras congeladas y a temperatura ambiente. Se determinó el método más eficiente. Se identificó de manera cualitativa la presencia de flavonoides y se cuantificó por ultravioleta la concentración de flavonoides totales expresados en quercetina. Los mejores resultados se obtuvieron para las muestras congeladas, empleando el método UVC. La cuantificación por ultravioleta permitió obtener concentraciones reproducibles en promedio de $178,4307 \mathrm{mg} / \mathrm{l}$ para los extractos congelados y 70,1590 mg/l para los extractos a temperatura ambiente, obtenidos de la envoltura de la cebolla roja.

Palabras clave: Centrifugación, polifenoles, quercetina, ultrasonido, vortex.

\begin{abstract}
The purpose of this work was to quantify total flavonoids, expressed in quercetin, of red onion outer covering. Onion samples were acquired from the peasant ecological market in Cali city (Valle del Cauca). Methanol extracts were performed by using shaking, ultrasound and UVC (ultrasound, vortex, and centrifugal). Frozen samples and room temperature were used. The most efficient method was determined. Presence of flavonoids was qualitatively identified, and total flavonoids concentration, expressed in quercetin, was quantified through ultraviolet. The best results were obtained for frozen samples, by using UVC method. Quantification through ultraviolet led to achieve reproducible concentrations in average of $178,4307 \mathrm{mg} / \mathrm{l}$, for frozen extracts, and 70,1590 $\mathrm{mg} / \mathrm{l}$ for room temperature extracts, obtained from red union covering.
\end{abstract}

Key words: Quercetin, polyphenols, ultrasound, vortex, centrifugation.
Recibido: 02/02/2016

Revisado: 15/02/2016

Aceptado: 01/12/2016

Correspondencia de autor: juan.sebastian.ramirez@ correounivalle.edu.co

(C) 2016 Universidad La Gran Colombia. Este es un artículo de acceso abierto, distribuido bajo los términos de la licencia Creative Commons Attribution License, que permite el uso ilimitado, distribución y reproducción en cualquier medio, siempre que el autor original y la fuente se acrediten.

\section{Cómo citar:}

Fajardo-Romero, A. Arroyo-Rivera, A. Ramírez-Navas, J.S. (2016) Extracción de flavonoides totales de la envoltura externa de cebolla roja (Allium cepa) UGCiencia 22, 119-126.

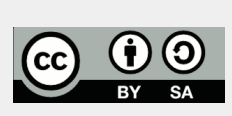




\section{Introducción}

La cebolla roja (Allium Cepa L.) es la segunda hortaliza más consumida a nivel mundial (Guerrero Riaño, Polo Mendoza, Villa Sarmiento, y Torres Jiménez, 2002). Ha sido utilizada como un recurso importante, tanto en el campo gastronómico, como en el médico y farmacéutico (Bystrická, Musilová, Vollmannová, Timoracká, y Kavalcová, 2013; Rose, Whiteman, Moore, y Zhu, 2005). Éstas han sido utilizadas como aditivos naturales para retrasar el deterioro de los alimentos (Navas, Carrasquero-Durán, y Flores, 2006). Por otra parte, hay evidencia de efectos benéficos para la salud, tales como antialérgico, antiinflamatorio, cardioprotector, vasodilatador, anticarcenogenic y propiedades antioxidantes (Shon, Choi, Kahng, Nam, y Sung, 2004).

Los flavonoides son metabolitos secundarios en las plantas, responsables de la pigmentación de las flores. Además de trayendo atraer agentes polinizadores y son protectores de los rayos ultravioleta. En la actualidad, se conoce su uso como antioxidante y antiinflamatorio (Jan y Badar, 2012). Las cebollas son ricas en flavonoides y en compuestos azufrados (Block, 1985; Lim, 2014). Posee una concentración de flavonoides superior con respecto a otros vegetales. Entre los principales se encuentran la quercetina, la isoramnetina y el kaempferol (Benítez García, 2011). Los derivados de la quercetina son los encargados de dar el color amarillo a la cebolla blanca, mientras que las antocianinas dan el color purpura a la cebolla roja. Una importante aplicación de la quercetina está en la inhibición de las acciones de lipooxigenasa donde produce una disminución de la formación de la ciclooxigenasa que es la encargada de los medidores de la inflamación (Lakhanpal y Kumar Rai, 2007). En variedades de cebollas doradas, el contenido de quercetina total cubre un rango muy amplio, desde $192 \mathrm{mg} /$ $\mathrm{kg}$ hasta $1516 \mathrm{mg} / \mathrm{kg}$ tejido fresco (Sgroppo). Y para las cebollas rojas, $1337 \pm 3 \mathrm{mg} / \mathrm{kg}$ (Torija, Matallana, y Chalup, 2013). En un estudio realizado en las centrales de Abastos de Colombia (Bogotá) se determinó que la cebolla cabezona blanca y roja son procedentes principalmente de las regiones de Boyacá y Cundinamarca. Los autores estimaron que $33 \%$ de envoltura externa es desechada, esto equivale a $10048 \mathrm{~kg} /$ día, con respecto a diferentes hortalizas como la mazorca, la yuca, el rábano, la coliflor y el ajo (Ospina Machado y Villamizar, 2003).

El uso del extracto genera nuevas alternativas de aplicación de la envoltura de la cebolla, por lo cual es preciso determinar el contenido de flavonoides totales expresados en quercetina. El objetivo de este trabajo fue cuantificar los flavonoides totales, expresados en quercetina, de la envoltura externa de la cebolla roja.

\section{Materiales y métodos}

\section{Recolección de muestras}

La investigación se realizó en los laboratorios de la Universidad Santiago de Cali, Colombia entre los años 2014 y 2015. Se recolectaron cebollas en el mercado campesino ecológico de Cali. Las cebollas recolectadas estaban certificadas por la Corporación Autónoma Regional del Valle del Cauca (CVC) como productos orgánicos. Se eligieron veinticuatro cebollas al azar sin tener en cuenta su tamaño. Se organizaron las cebollas en grupos de seis. Se les retiró la cascará y se pesaron aproximadamente $15 \mathrm{~g}$ cáscara por cada conjunto para conformar seis muestras. Tres muestras se congelaron durante 24 horas a $-8^{\circ} \mathrm{C}$. Tres muestras se mantuvieron a temperatura ambiente. 


\section{Extracción de flavonoides por agitación}

Se siguió la metodología propuesta por Quiroz Reyes y Mendez Aguilar (2013), con algunas modificaciones en la cantidad de muestra y solvente; además del uso exclusivo de metanol y no de acetona. Para la extracción por agitación se realizó una extracción orgánico-ácida de las cáscaras de cebolla roja. Para ello, aproximadamente $15 \mathrm{~g}$ de cada muestra se extrajo con $200 \mathrm{ml}$ de solución acuosa de metanol $(50 \% \mathrm{v} / \mathrm{v})$ conteniendo $0,1 \mathrm{ml}$ de $\mathrm{HCl}$ $5 \%$. Se agitó con magneto durante una hora a temperatura ambiente. Posteriormente, se centrifugó durante quince minutos a $400 \mathrm{rpm}$. Los sobrenadantes se separaron y los residuos se extrajeron de nuevo con $200 \mathrm{ml}$ de solución acuosa de metanol (50 \% v/v), repitiendo la centrifugación y combinando los sobrenadantes con los obtenidos anteriormente.

\section{Extracción de flavonoides por ultrasonido}

Se siguió la metodología propuesta por Quiroz Reyes y Mendez Aguilar (2013), con algunas modificaciones. Para la extracción orgánico-ácida de la cáscara de cebolla roja por ultrasonido se pesaron aproximadamente 15 gramos de cada muestra y se extrajeron con 200 $\mathrm{ml}$ de solución acuosa de metanol $(50 \% \mathrm{v} / \mathrm{v})$ que contenía $0,1 \mathrm{ml}$ de $\mathrm{HCl} 5 \%$. Se colocaron a temperatura ambiente durante quince minutos en un aparato ultrasonido a $25 \mathrm{kHz}$, posteriormente tras centrifugar (quince minutos a $400 \mathrm{rpm}$ ) se separan los sobrenadantes y los residuos; se extrajo de nuevo con $200 \mathrm{ml}$ de solución acuosa de metanol $(50 \% \mathrm{v} / \mathrm{v})$ y se realizó nuevamente el proceso de extracción vía ultrasónica durante quince minutos, después se centrifugó y se combinaron los sobrenadantes con los obtenidos anteriormente.
Extracción de flavonoides por UVC (ultrasonido, vortex y centrifuga)

Se extrajeron $15 \mathrm{~g}$ de cáscara con $200 \mathrm{ml}$ de solución metanólica $50 \%$ conteniendo $0,1 \mathrm{~mL}$ de $\mathrm{HCl} 5 \%$ por diez minutos de sonicación. La mezcla se agitó con vórtex durante cinco minutos, se sometió a ultrasonido y se agitó una vez más, y se centrifugó durante diez minutos a $400 \mathrm{rpm}$ (Ko, Nile, Sharma, Li, y Park, 2014). Esta etapa se repitió dos veces, y los sobrenadantes se recogieron en un frasco de $400 \mathrm{ml}$.

Cuantificación de flavonoides totales por espectroscopia ultravioleta

Estos metabolitos se cuantificaron con cloruro de aluminio en metanol $50 \%$ a $374,5 \mathrm{~nm}$. Los resultados se expresaron como quercetina en una curva de calibración con concentraciones de 0 a 7,5 ppm (Ochoa Pacheco, Marín Morán, Rivero Breff, y Aguilera Saborít, 2013). En un volumen de $50 \mathrm{ml}$ se añadieron: $1 \mathrm{ml} \mathrm{de}$ extracto de muestra extraída y $1 \mathrm{ml}$ del cloruro de aluminio al $5 \%$ en metanol, se agitó y se dejó reposar por diez minutos. Se enrasó con metanol $50 \% \mathrm{v} / \mathrm{v}$ y se realizó la medición a $374,5 \mathrm{~nm}$.

\section{Análisis cualitativo (Prueba de Shinoda)}

Se tomó en un tubo de ensayo $1 \mathrm{ml}$ de extracto obtenido por el método UVC. Se agregaron algunas limaduras de magnesio. Se adicionaron cuidadosamente por la pared del tubo unas gotas de $\mathrm{HCl}$ concentrado. La aparición de coloración roja comprueba positivamente la existencia de flavonoides (Carrión Jara y García Gómez, 2010). 


\section{Análisis estadístico}

Los datos obtenidos se analizaron empleando el paqueteestadísticoSPSS(IBMStatistics.v.18.0), por medio de análisis de varianza ANOVA se evaluaron las diferencias significativas entre los tratamientos. Como análisis post hoc se utilizó el test Tuckey con $\alpha$ de 0,05 .

\section{Resultados}

De los tres métodos de extracción de quercetina, el UVC (ultrasonido, vortex y centrifuga) fue el mejor para la extracción al presentar mayor concentración del metabolito de interés, aunque requirió de mayor tiempo de extracción.
Permitió combinar las tres técnicas de contacto de la muestra con el solvente para una mejor extracción. La cuantificación por ultravioleta obtuvo concentraciones reproducibles.

En la Figura 1 se presenta la curva de calibración usando como estándar quercetina. Al realizar el barrido espectral se encontró que la máxima longitud de onda fue de $374,5 \mathrm{~nm}$. Se trabajó la curva de calibración a esta longitud de onda, tomando lectura de siete soluciones de quercetina preparadas con poca anterioridad por su inestabilidad a la luz. Posteriormente, se leyeron las dieciocho muestras a las condiciones de temperatura y los distintos métodos.

Figura 1. Curva de calibración por ultravioleta y ecuación de la recta, usando como estándar quercetina, para compararar los resultados obtenidos de las extracciones de flavonoides totales y concentración de quercetina en cebolla roja

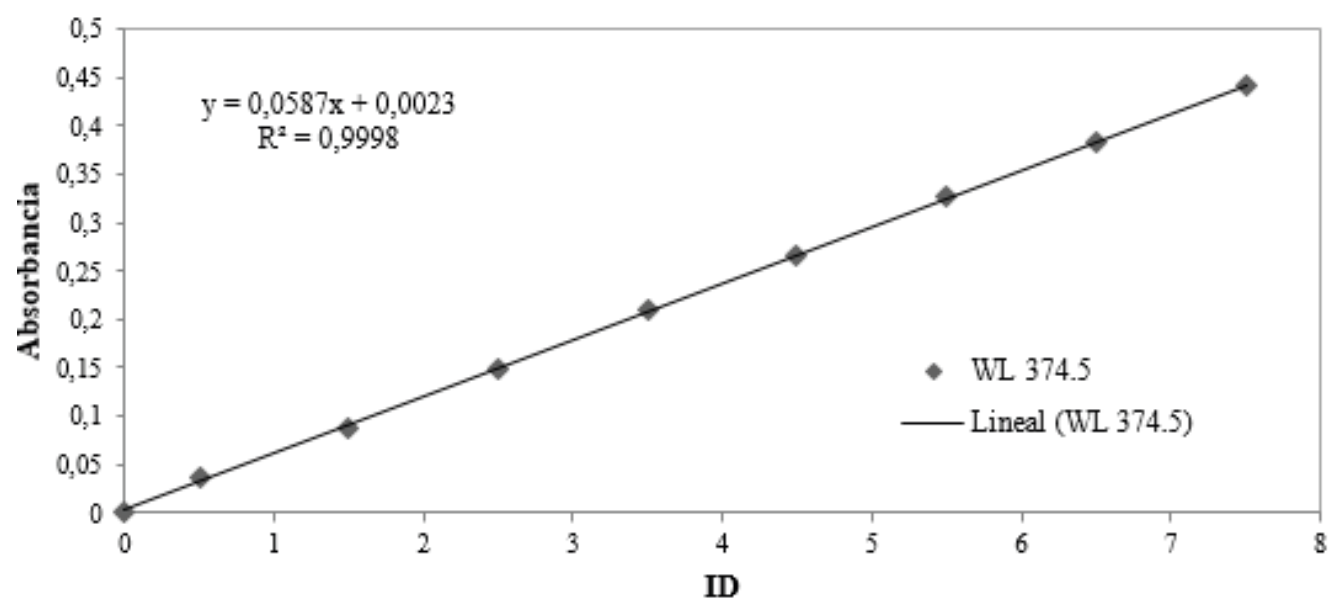

En la Figura 2 se presentan las concentraciones de quercetina (ppm) obtenidas. Se observa que el el método UVC, permitió obtener las mayores concentraciones. En las muestras que fueron previamente congeladas, se obtuvieron concentraciones más altas que aquellas que se dejaron a temperatura ambiente. Esto se debe a que el proceso de congelación empleado fue lento, generando cristales de hielo grandes, que rompen la pared celular. La ruptura de la pared celular permite extraer con mayor eficiencia el líquido intracelular (Millán et al., 2007).

Valor obtenido del promedio de tres réplicas de extractos de cáscara de cebolla. ${ }^{\mathrm{a}, \mathrm{b}, \mathrm{c}, \mathrm{d}, \mathrm{e}}$ Los resultados en columnas que tienen superíndices iguales no difieren significativamente $(p<0,05)$. 
Figura 2. Concentraciones de quercetina en cebolla roja, obtenidas por los tres métodos de extracción, por agitación, ultrasonido y por UVC (ultrasonido, vortex y centrifuga).

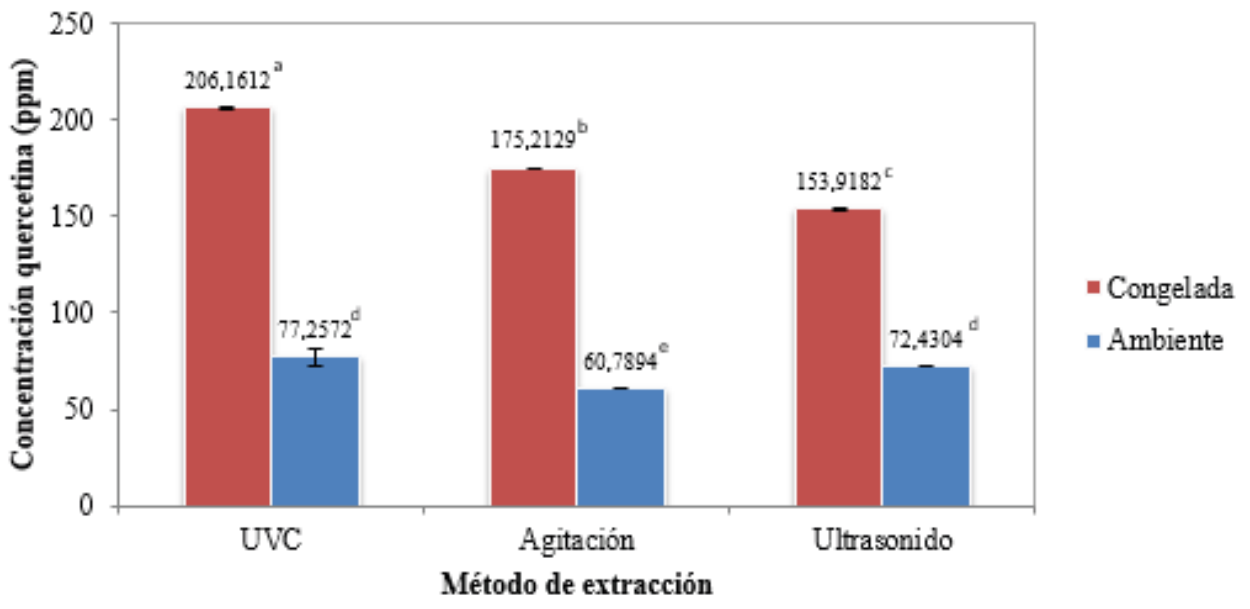

Adicionalmente, se realizó un análisis de varianza encontrando diferencias significativas para las condiciones de temperatura. De igual manera existieron diferencias significativas para todos los métodos en las muestras congeladas. Por lo contrario, no hubo diferencia significativa entre los resultados de los métodos de ultrasonido y UVC para las muestras dejadas a temperatura ambiente.

El análisis estadístico permitió seleccionar el método UVC con muestras congeladas, para la extracción de los flavonoides totales de la cáscara de la cebolla roja. Entre las ventajas evaluadas están la mayor concentración de flavonoides totales expresados en quercetina, mayor extracción y mejor rendimiento del extracto obtenido, y menor generación de residuos de solvente.

\section{Discusión de resultados}

Ultrasonido: los resultados obtenidos de los diferentes métodos de extracción fueron coherentes respecto a las técnicas que empleadas. En el método de ultrasonido, las cáscaras congeladas dieron una concentración menor comparado con el método de agitación y UVC. Esto se debe a que el equipo se utilizó a una intensidad alta, aumentando la temperatura y generando burbujas de cavitación que degradan los flavonoides. Por esta razón no se realizó una adecuada extracción. Para mejorar la extracción, el método requiere menos tiempo de extracción, a menor intensidad que la empleada en este caso y menos cantidad de solvente.

El ultrasonido se utilizó por la capacidad de extracción de compuestos orgánicos de matrices líquidas y sólidas, gracias a la eficiencia del contacto entre la cáscara de la cebolla y el metanol, como resultado se logró reducir el tiempo de extracción, minimizar el volumen de muestra como el volumen de solvente utilizado. La extracción es exitosa por sus burbujas de cavitación, estas son formadas por el rompimiento del líquido y dan origen a burbujas de gas (cavidades) que crecen y posteriormente colapsan (implotan) a una velocidad mayor del sonido, emitiendo ondas de choque (ondas de presión abrupta) (Morales, 2011). 
Agitación: el método por agitación dio un mejor resultado en comparación con ultrasonido, en las muestras congeladas, debido al movimiento constante que se emitió y a que la temperatura estaba controlada. Este método permite que cantidades de solvente, pobre en los compuestos de interés, entren en contacto con la muestra y se alcance un nuevo equilibrio de saturación. El uso de agitadores mecánicos, desplaza el equilibrio en el sentido de la saturación del solvente, aumentando la eficiencia del proceso (Jiménez, 2008) ${ }^{\circ} \mathrm{C}$.

\section{UVC}

El método UVC (combinación de ultrasonido vortex y centrifuga) empleado para las muestras congeladas y ambiente; dio como resultado una mayor concentración de quercetina para las muestras congeladas en comparación con las muestras a temperatura ambiente debido a la unión de los tres métodos y las diferentes condiciones de temperatura que se usó en ambos casos, favoreciendo en las muestras congeladas la formación de cristales de hielo. En la Figura 2 se observan que este método fue el adecuado porque la unión de tres equipos de extracción no convencionales optimizó el proceso de extracción de flavonoides.

El vortex tiene un movimiento vibratorio generando ondas longitudinales, así el extracto va a girar en forma de vórtice y en la misma dirección en la que las ondas se propagan ayudando a un mejor contacto del solido con el solvente. En el ultrasonido el método del vortex también disminuye el tiempo y homogeniza la muestra. Finalmente, el uso de la centrífuga fue usado para separar los sedimentos que hayan quedado de los otros dos métodos de extracción, además este proceso ayudó a homogenizar el extracto y así retirar cualquier sedimento existente.
Si no se posee algunos de los equipos del UVC, la extracción podría emplearse el método de agitación, manteniendo una velocidad constante y una temperatura controlada. Empleando un volumen de solvente de $400 \mathrm{ml}$ y un tiempo de dos horas. La diferencia de los dos métodos varía en la cantidad de solvente utilizado, haciendo que el método de agitación sea más contaminante por la generación de residuos.

\section{Reacción de Shinoda}

Los extractos obtenidos por el método UVC dieron resultado positivo a la prueba de Shinoda generando una coloración roja como respuesta positiva. En la reacción de Shinoda (Figura 3), el magnesio metálico es oxidado por el $\mathrm{HCl}$ concentrado, dando como productos al $\mathrm{H}_{2}$, que es eliminado en forma de gas y el $\mathrm{MgCl}_{2}$, que es el que forma complejos con los flavonoides dando coloraciones características. El magnesio divalente intensifica la coloración por estar doblemente coordinado. Donde el magnesio metálico es oxidado por el $\mathrm{HCl}$ concentrado, dando como productos al $\mathrm{H}_{2}$, que es eliminado en forma de gas y el $\mathrm{MgCl}_{2}$, que es el que forma complejos con los flavonoides dando coloraciones características.

Figura 3. Reacción de Shinoda
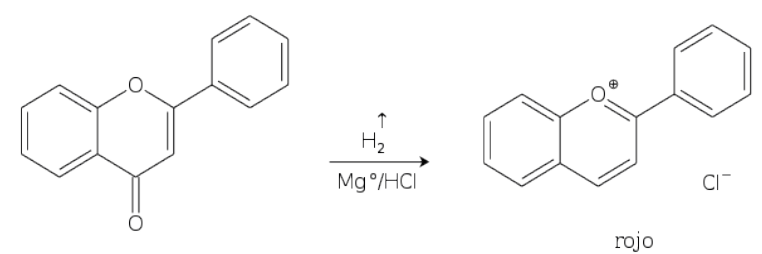

\section{Conclusiones}

Se emplearon tres métodos de extracción de quercetina como estudio preliminar. Siendo el método UVC (ultrasonido, vortex y centrifuga) el idóneo para la extracción al presentar mayor 
concentración del metabolito de interés, aunque requirió de mayor tiempo de extracción, este permitió combinar las tres técnicas de contacto de la muestra con el solvente para una mejor extracción. La cuantificación por ultravioleta permitió obtener concentraciones reproducibles.

\section{Referencias bibliográficas}

Benítez García, V. (2011). Caracterización de subproductos de cebolla como fuente de fibra alimentaria y otros compuestos bioactivos. Doctorado, Universidad Autónoma de Madrid, Madrid, España. Recuperado de http://hdl.handle. net/10261/101601

Block, E. (1985). Química del ajo y de la cebolla. [Científico ]. Investigación y Ciencia, 104, 98 - 104.

Bystrická, J., Musilová, J., Vollmannová, A., Timoracká, M., y Kavalcová, P. (2013). Bioactive components of onion (Allium cepa L.) - a Review. Acta Alimentaria, 42(1), 11-22. doi: http://dx.doi. org/10.1556/AAlim.42.2013.1.2

Carrión Jara, A. V., y García Gómez, C. R. (2010). Preparación de extractos vegetales: determinación de eficiencia de metódica. Bioquímica Farmacéutica, UniversidaddeCuenca,Cuenca,Ecuador. Recuperado de http://dspace.ucuenca. edu.ec/handle/123456789/2483

Guerrero Riaño, E., Polo Mendoza, E., Villa Sarmiento, G., y Torres Jiménez, O. (2002). Capacitación a pequeños productores de los municipios de Repelón y Santa Lucia en el manejo técnico-empresarial del cultivo de cebolla cabezona bajo condiciones de riego. http://www.agronet.gov.co/www/ docs_si $2 /$ cartilla $\% 20$ didactica $\% 20 \mathrm{de} \% 20$ cebolla\%20cabezona.pdf
Jan, M., y Badar, A. (2012). Effect of crude extract of Raphanus sativus roots on isolated trachea of albino rat. Pakistan Journal of Physiology, 8(1), 23-26.

Jiménez, A. (2008). Desarrollo de un proceso a escala de laboratorio para la extracción de colorante a partir de la flor del árbol de majagua (hibiscus elatus). Universidad EAFIT Medellín. Recuperado de https://repository.eafit. edu.co/bitstream/handle/10784/352/ AstridEliana_JimenezRamirez_2008. pdf? sequence $=1$ \&isAllowed $=y$

Ko, E. Y., Nile, S. H., Sharma, K., Li, G. H., y Park, S. W. (2014). Effect of different exposed lights on quercetin and quercetin glucoside content in onion (Allium cepa L.). Saudi Journal of Biological Sciences, 22(1), 398-403.

Lakhanpal, P., y Kumar Rai, D. (2007). Quercetin: A versatile flavonoid. Journal of Medical Update, 2(2), 22-37.

Lim, T. K. (2014). Edible Medicinal and Non Medicinal Plants: Modified Stems, Roots, Bulbs (Vol. 9). Netherlands: Springer.

Morales, M. (2011). Determinación de hidrocarburos en muestras de agua por extracción líquido-líquido asistida por ultrasonido. Universidad de Alicante Alicante. Recuperado dehttp://iuaca.ua.es/ es/master-agua/documentos/-gestadm/ trabajos - fin-d e - mas ter $/$ maria-fernanda-morales.pdf

Navas, P. B., Carrasquero-Durán, A., y Flores, I. (2006). Effect of black tea, garlic and onion on corn oil stability and fatty acid composition under accelerated oxidation. International Journal of Food Science \& Technology, 41(3), 243-247. 
Ochoa Pacheco, A., Marín Morán, J., Rivero Breff, D., y Aguilera Saborít, E. M. (2013). Caracterización física y química de extractos totales de petiveria alliacea l. con acción antimicrobiana. Revista Mexicana de Ciencias Farmacéuticas, 44(1), 52-59.

Ospina Machado, J. E., y Villamizar, F. (2003). Evaluación de residuos y daños en hortalizas de alto consumo en centrales de abastos en Colombia. Revista Iberoamericana de Tecnología Postcosecha, 5(1), 1-7.

Quiroz Reyes, y Mendez Aguilar. (2013). Nanoencapsulación de flavonoides procedentes de grano de cacao (Theobroma cacao L.). VI Simposio de Tecnología Avanzada. http://www. repositoriodigital.ipn.mx/bitstream/ handle/123456789/11250/2010\%20 VI\%20STA \%2018-19.pdf?sequence=1

Rose, P., Whiteman, M., Moore, P. K., y Zhu, Y. Z. (2005). Bioactive S-alk(en)yl cysteine sulfoxide metabolites in the genus Allium: the chemistry of potential therapeutic agents. Natural Product Reports, 22(3), 351-368. doi: http:// dx.doi.org/10.1039/B417639C

Sgroppo, S. d. A., B Cano, MP Avanza, Jorge R. Modificaciones del contenido en quercetina en cebollas por accion de microondas.

Shon, M.-Y., Choi, S.-D., Kahng, G.-G., Nam, S.-H., y Sung, N.-J. (2004). Antimutagenic, antioxidant and free radical scavenging activity of ethyl acetate extracts from white, yellow and red onions. Food and Chemical Toxicology, 42(4), 659-666.
Torija, M. E., Matallana, M. C., y Chalup, N. (2013). El ajo y la cebolla: de las medicinas antiguas al interés actual. Bol. R. Soc. Esp. Hist. Nat. Sec. Biol., 107(1), 29-37. 